\title{
The relationship among cardiac structure, dietary salt and aldosterone in patients with primary aldosteronism
}

\author{
Chi-Sheng Hung ${ }^{1}$, Xue-Ming Wu ${ }^{2}$, Ching-Way Chen ${ }^{3}$, Ying-Hsien Chen ${ }^{1}$, Vin-Cent \\ $\mathbf{W u}^{1}$, Che-Wei Liao ${ }^{4}$, Yi-Yao Chang ${ }^{5}$, Ruh-Fang Yen ${ }^{6}$, Ching-Chu Lu${ }^{6}$, Mao-Yuan $M$. \\ Su${ }^{7}$, Kao-Lang Liu' ${ }^{7}$ Chin-Chen Chang7, Li-Yu Daisy Liu ${ }^{8}$, Kwan-Dun Wu ${ }^{1}$, Yen-Hung \\ Lin ${ }^{1}$ and the TAIPAI Study Group \\ ${ }^{1}$ Department of Internal Medicine, National Taiwan University Hospital and National Taiwan University College of Medicine, \\ Taipei, Taiwan \\ ${ }^{2}$ Department of Internal Medicine, Taoyuan General Hospital, Taoyuan, Taiwan \\ ${ }^{3}$ Department of Internal Medicine, National Taiwan University Hospital Yun-Lin Branch, Yun-Lin, Taiwan \\ ${ }^{4}$ Department of Internal Medicine, National Taiwan University Hospital Hsin-Chu Branch, Hsin-Chu, Taiwan \\ ${ }^{5}$ Cardiology Division of Cardiovascular Medical Center, Far Eastern Memorial Hospital, New Taipei City, Taiwan \\ ${ }^{6}$ Department of Nuclear Medicine, National Taiwan University Hospital and National Taiwan University College of Medicine, \\ Taipei, Taiwan \\ ${ }^{7}$ Department of Medical Imaging, National Taiwan University Hospital and National Taiwan University College of Medicine, \\ Taipei, Taiwan \\ ${ }^{8}$ Department of Agronomy, Biometry Division, National Taiwan University, Taipei, Taiwan \\ Correspondence to: Yen-Hung Lin, email: austinr34@gmail.com
}

Keywords: primary aldosteronism, salt intake, left ventricular mass, TAIPAI

Received: December 08, $2016 \quad$ Accepted: April 05, 2017 Published: April 28, 2017

Copyright: Hung et al. This is an open-access article distributed under the terms of the Creative Commons Attribution License 3.0 (CC BY 3.0), which permits unrestricted use, distribution, and reproduction in any medium, provided the original author and source are credited.

\section{ABSTRACT}

Salt intake is highly associated with cardiac structure in patients with primary aldosteronism (PA). We investigated the association among dietary salt intake, aldosterone and left ventricular mass in patients with PA. We enrolled 158 patients with PA and 158 patients with essential hypertension. We measured 24-hour urinary sodium (UNa) and aldosterone (UAldo) level and echocardiography parameters. In patients with PA, the UAldo level was positively correlated with left ventricular mass index ( $L V M I ; r=0.231, p=0.007)$. The UNa level was not linearly correlated with left ventricular structural parameters in patients with PA. To test if UNa has a non-linear relationship with LVMI among patients with PA, we categorized the participants according to the tertile of UNa (low, median, and high tertile). PA patients with medium tertile of UNa had significant lower LVMI than the other two groups (LVMI: $144.1 \pm 42.9,121.1 \pm 33.4$, and $136.7 \pm 32.8$ $\mathrm{g} / \mathrm{m} 2$, from the lowest to the highest tertile of Una; analysis of variance $\mathrm{p}=0.006$, post-hoc $p<0.05$ ). Multifactor analysis of variance confirmed this finding after adjustment for clinical parameters. Post-hoc analyses revealed that the high UNa tertile was associated with higher left ventricular end-diastolic volume compared with medium UNa tertile; while the low UNa tertile was associated with higher mean wall thickness compared with medium UNa tertile. The findings imply the reasons for increased LVMI may be different in patients with the highest and lowest UNa tertile. In conclusion, the medium tertile of 24-hour UNa is associated with lowest LVMI in patients with PA. 


\section{INTRODUCTION}

Primary aldosteronism (PA), characterized by excessive production of aldosterone, is now considered to be one of the main causes of secondary hypertension [1]. The reported prevalence of PA in patients with hypertension ranges from 5-15\% [2]. Patients with PA have high rates of cardiovascular complications, including left ventricular hypertrophy (LVH), heart failure, myocardial infarction, stroke and atrial fibrillation $[3,4]$.

$\mathrm{LVH}$, or increased LV mass, is more common in patients with PA than in those with essential hypertension (EH) or other types of secondary hypertension [5]. LVH develops early in the course of PA, and is preceded by other end-organ damage [6]. LVH is also an independent risk factor for cardiovascular mortality [7]. Patients with PA have a higher rate of $\mathrm{LVH}$ and an inappropriately high LV mass for the degree of workload and blood pressure compared to patients with $\mathrm{EH}$ [8]. Aldosterone has been reported to be a major cause of inappropriate increases in LV mass in patients with PA, and has been shown to directly cause cardiac structural damage, including cardiomyocyte hypertrophy and myocardial fibrosis, independently of the effect of blood pressure or angiotensin II $[9,10]$. Rossi et al reported 323 patients with PA, in whom the LV mass improved to the level of optimally treated $\mathrm{EH}$ after adrenalectomy or treatment with a mineralocorticoid receptor antagonist during longterm follow-up [11].

In patients with $\mathrm{EH}$, dietary sodium intake and plasma aldosterone concentration have been reported to be independently associated with LV mass $[12,13]$. In patient with PA, however, the relative contributions of sodium and aldosterone to LVH are less clear. A recent study on patients with PA suggested a positive correlation between dietary sodium and LV mass [14], although the patients with PA in their study had relatively high salt intake compared to the average daily salt intake worldwide [15], and it is unclear whether the same results would have been found in PA patients with normal or low sodium intake. Moreover, a large clinical observation study reported a U-shaped relationship between sodium intake and mortality [16]. A recent animal study also reported that lower salt intake could still induce LV hypertrophy and fibrosis in the presence of excess aldosterone [17]. Hence, it is possible that the relationship between sodium intake and LV mass is nonlinear.

We hypothesized that in patients with PA, the relationship between salt intake and LV mass would be weakest in those with a medium sodium intake, and that the relationship between aldosterone and LV mass would be positive and linear. We conducted this crosssectional study to evaluate the relationships between 24 hour urinary aldosterone and sodium excretion and LV structural parameters using echocardiography.

\section{MATERIALS AND METHODS}

\section{Patients}

This cross-sectional study enrolled 158 patients diagnosed with PA from October 2006 to March 2010, all of whom were registered in the Taiwan Primary Aldosteronism Investigation (TAIPAI) database. This database was constructed for quality assurance in one medical center (National Taiwan University Hospital, Taipei, Taiwan), and its three affiliated hospitals around Taiwan (National Taiwan University Hospital YunLin branch, Yun-Lin, southern Taiwan; Far-Eastern Memorial Hospital, Taipei; and Taoyuan General Hospital, Taoyuan, central Taiwan) [18]. Another 158 patients with EH were enrolled for comparison. The medical history of the subjects, including demographic data and current medications, was recorded. The diagnosis of EH was made by exclusion according to standard algorithms based on clinical history, and biochemical investigations of all detectable forms of secondary hypertension. All of the patients were evaluated with echocardiography and 24-hour urine collection for sodium and aldosterone concentrations. The 24-hour urine collection was performed under the subjects' usual diet reflecting salt appetite. This study complied with the Declaration of Helsinki and was approved by the Institutional Review Board of National Taiwan University Hospital (Taipei, Taiwan). Informed consent was obtained from all participants before inclusion in the study.

\section{Laboratory measurements}

A 24-hour urine sample with the first urine of the day included was collected from all subjects and refrigerated until analysis. The 24-hour urinary sodium and aldosterone amount (UNa and UAldo, respectively) were calculated by multiplying the urine sodium or aldosterone concentration by the daily urine amount. When interpreting the results of 24-hour urine collection, we assessed the adequacy of collection by quantifying the 24-hour urine creatinine excretion value, defined as a value between 15 and 20 $\mathrm{mg} / \mathrm{kg}$ of body weight. The urine samples were stored in plastic containers at $4^{\circ} \mathrm{C}$.

The plasma aldosterone concentration (PAC) was measured using radioimmunoassays with commercial kits (Aldosterone Maia Kit, Adaltis Italia S.P.A., Bologna, Italy) [19]. Plasma renin activity (PRA) was calculated as the generation of angiotensin-I in vitro using a commercially available radioimmunoassay kit (Cisbio, Bedford, MA). All antihypertensive medications were discontinued for at least 21 days before measuring plasma PRA and PAC. Diltiazem and/or doxazosin was administered to control markedly high blood pressure when required. 


\section{Diagnostic criteria for PA}

Fulfillment of the following three conditions was taken to confirm a diagnosis of aldosteronism: (1) autonomous excess aldosterone production evidenced with an aldosterone to renin ratio (ARR) $>35$; (2) a TAIPAI score $>60 \%$ [20]; (3) post-saline loading PAC $>10 \mathrm{ng} /$ $\mathrm{dl}$, or PAC/PRA $>35(\mathrm{ng} / \mathrm{dL}) /(\mathrm{ng} / \mathrm{mL} / \mathrm{h})$ shown in a post captopril/losartan test, or PAC $>6 \mathrm{ng} / \mathrm{dL}$ as indicated by a fludrocortisone suppression test [21].

\section{PA subtype identification}

Aldosterone-producing adenoma was identified on the basis of the following four conditions: (1) autonomous excess aldosterone production evidenced with an ARR > 35, a TAIPAI score $>60 \%$ [20], and post-saline loading PAC $>10 \mathrm{ng} / \mathrm{dl}$; (2) adenoma evidenced on a computed tomography (CT) scan for pre-operative evaluation; (3) lateralization of aldosterone secretion at adrenal vein sampling or during dexamethasone suppression NP-59 SPECT/CT [22]; (4) pathologically proven adenoma after adrenalectomy for the patients who underwent surgery, and the subsequent emergence of either a cure pattern of hypertension without anti-hypertensive agents or improvement in hypertension, potassium, PAC, and PRA $[21,23,24]$.

Idiopathic hyperaldosteronism was defined on the basis of the following four criteria: (1) autonomous ex-cess aldosterone production evidenced with an ARR $>35$, a TAIPAI score $>60 \%$ [20], and post-saline loading PAC $>10 \mathrm{ng} / \mathrm{dl}$; (2) evidence of bilateral diffuse enlargement as seen on a CT scan for pre-operative evaluation; (3) non-lateralization of aldosterone secretion at AVS or during dexamethasone suppression NP-59 SPECT/CT [22]; (4) evidence of diffuse cell hyperplasia reported in following pathology studies for those who underwent surgery.

\section{Echocardiography}

All echocardiography was performed using a Hewlett-Packard 5500 ultrasound system with an S3 transducer (1.0-3.0 $\mathrm{MHz})$. Transthoracic echocardiographic images were obtained in fundamental imaging modes. Two-dimensional, M-mode, Doppler and tissue Doppler ultrasonography were performed in each patient, and the dimensions of the chamber, wall thickness and left ventricular ejection fraction (M-mode) were measured according to the guidelines of the American Society of Echocardiography [25].

LVMI was derived from echocardiography according to the formula reported by Devereux and Reichek: $[\mathrm{LV}$ mass $=1.04 \times[$ (septal thickness $+\mathrm{LV}$ end-diastolic diameter + posterior wall thickness $)^{3}-(\mathrm{LV}$ end-diastolic diameter) ${ }^{3}$ ]-13.6] [26]. Predicted LVMI was estimated using a previously derived equation: predicted $\mathrm{LVM}=55.37+6.64 \times$ height $^{2.7}+0.64 \times$ stroke work-
$18.07 \times$ gender (where gender was scored as male $=1$ and female=2) [27]. Left ventricle volume was calculated using Tericholz's formula, and stroke work was calculated as systolic blood pressure (in $\mathrm{mmHg}$ ) x stroke volume $\mathrm{x} 0.0144$ [8]. Inappropriate LVMI or excessive LV mass compared to the predicted LVMI, was defined as: measured LVMI - predicted LVMI. LVH was defined according to Devereux's criteria: LVMI $\geq 134 \mathrm{~g} / \mathrm{m}^{2}$ in men and $110 \mathrm{~g} / \mathrm{m}^{2}$ in women [28].

\section{Statistical analysis}

All continuous variables were expressed as mean \pm standard deviation if normally distributed. Nonnormally distributed variables were expressed as median and interquartile range. Comparison of continuous variables between the two groups were preformed using the Student's t-test (normally distributed) or Wilcoxon rank sum test (non-normally distributed). Differences between proportions were assessed using the chi-square test. Urinary aldosterone concentration was log-transformed before the correlation study due to non-normality, which was determined by the Kolmogorov-Smirnov test.

Pearson's correlation tests were first used to analyze the correlations between LVMI and 24-hour UAldo or UNa level. Partial correlations were then used to adjust for age, gender and systolic blood pressure. To test the non-linear relationship between urinary aldosterone or sodium and LVMI, we categorized the patients into tertiles according to the UAldo or UNa level (low, medium, and high tertiles). Multifactor ANOVA was performed to test the effect of UAldo or UNa tertile on LVMI, with adjustments for age, sex, mean blood pressure and duration of hypertension. An interaction term of UAldo and UNa was included to test the interaction. According to the LVMI formula, LVMI was determined by both LV wall thickness and end-diastolic volume. Therefore, if the association between UAldo or UNa and LVMI was significant, we further analyzed the association between UAldo or UNa and wall thickness and end-diastolic volume.

ANOVA was used separately for the patients with PA and those with EH. Tukey's honest significant difference test was used for post-hoc analysis. All statistical analyses were performed using Stata/SE software version 11.2 for Windows (StataCorp LP, Texas) and R 2.14.0 software (R Foundation for Statistical Computing, Vienna, Austria).

\section{RESULTS}

One hundred and fifty-eight PA patients and 158 EH patients were enrolled in this study. The mean age of all participants was $51.8 \pm 12.4$ years, and the baseline clinical characteristics are shown in Table 1. The mean 
Table 1: Baseline characteristics and echocardiographic findings of study participants

\begin{tabular}{|c|c|c|c|}
\hline & $\begin{array}{l}\text { Primary aldosteronism } \\
\qquad(\mathrm{n}=158)\end{array}$ & $\begin{array}{l}\text { Essential hypertension } \\
\quad(\mathbf{n}=158)\end{array}$ & P value \\
\hline Age, year, mean(SD) & $51.8(11.6)$ & $51.8(13.1)$ & 0.978 \\
\hline Sex, male, $n(\%)$ & $68(43 \%)$ & $87(55.1 \%)$ & 0.033 \\
\hline Body mass index, kg/m², mean(SD) & $25.1(3.5)$ & $26.8(12.1)$ & 0.096 \\
\hline Duration of hypertension, year, mean(SD) & $8.5(7.8)$ & $6.2(7.1)$ & 0.007 \\
\hline Systolic blood pressure, mmHg, mean(SD) & $151.8(20.6)$ & 145.1(21.6) & 0.005 \\
\hline Diastolic blood pressure, mmHg, mean(SD) & $89.3(12.5)$ & $86.7(13.0)$ & 0.075 \\
\hline APA, n(\%) & $124(79.0 \%)$ & - & - \\
\hline \multicolumn{4}{|l|}{ Medications } \\
\hline ARB or ACEI, n(\%) & $24(15.1 \%)$ & $20(12.7 \%)$ & 0.472 \\
\hline Calcium channel blocker & $104(67.1 \%)$ & $91(58.0 \%)$ & 0.115 \\
\hline Thiazide, $\mathrm{n}(\%)$ & $62(39.2 \%)$ & $17(10.8 \%)$ & $<0.001$ \\
\hline Beta-blocker, n(\%) & $73(47.1 \%)$ & $36(22.8 \%)$ & $<0.001$ \\
\hline Alpha-blocker, n(\%) & $33(21.3 \%)$ & $23(14.6 \%)$ & 0.120 \\
\hline Direct vasodilator, $\mathrm{n}(\%)$ & $4(2.6 \%)$ & $7(4.4 \%)$ & 0.374 \\
\hline \multicolumn{4}{|l|}{ Biochemistry data } \\
\hline Serum sodium, mEq/L, mean(SD) & $139.9(8.2)$ & $139.8(2.2)$ & 0.892 \\
\hline Serum potassium, mEq/L, mean(SD) & $3.5(0.8)$ & $4.2(0.4)$ & $<0.001$ \\
\hline $\begin{array}{l}\text { Plasma aldosterone concentration*, ng/dL, } \\
\text { median }\left(25^{\text {th }}, 75^{\text {th }} \text { percentile }\right)\end{array}$ & $41.4(28.6,63.2)$ & $35.3(24.6,49.9)$ & $<0.001$ \\
\hline $\begin{array}{l}\text { Plasma renin activity* }, \mathrm{ng} / \mathrm{ml} . \mathrm{hr}, \text { median } \\
\left(25^{\text {th }}, 75^{\text {th }} \text { percentile }\right)\end{array}$ & $0.27(0.07,0.64)$ & $2.36(1,7.48)$ & $<0.001$ \\
\hline $\begin{array}{l}\text { Aldosterone to renin ratio* } \text {, median }\left(25^{\text {th }}, 75^{\text {th }}\right. \\
\text { percentile })\end{array}$ & $141.6(50.3,683.5)$ & $12.9(6.4,45.2)$ & $<0.001$ \\
\hline $\begin{array}{l}\text { Urinary aldosterone* } \mu \mathrm{g} / 24 \text { hours, median } \\
\left(25^{\text {th }}, 75^{\text {th }} \text { percentile }\right)\end{array}$ & $13.76(7.1,27.5)$ & $8.7(5.5,15.3)$ & $<0.001$ \\
\hline Urinary sodium, mmol/24 hours, mean(SD) & 154.4(91.8) & $153.8(70.6)$ & 0.946 \\
\hline $\begin{array}{l}\text { Glomerular filtration rate }\left(\mathrm{ml} / \mathrm{min} / 1.73 \mathrm{~m}^{2} \text {, }\right. \\
\text { by MDRD equation), mean(SD) }\end{array}$ & $85.4(25.9)$ & $84.7(25.6)$ & 0.809 \\
\hline
\end{tabular}

ACEI: angiotensin converting enzyme inhibitor; ARB: angiotensin II Receptor Blocker; MDRD: Modification of Diet in Renal Disease

*Expressed as mean and interquartile range

UNa level was $154.1 \pm 82.1 \mathrm{mmol} /$ day in all participants $(154.4 \pm 91.8 \mathrm{mmol} / \mathrm{day}$ in the PA group and $153.8 \pm 70.6$ $\mathrm{mmol} /$ day in the EH group). The median 24-hour UAldo level was 13.76 and $8.7 \mu \mathrm{g}$ per day in the PA and $\mathrm{EH}$ groups, respectively. Patients with PA had a higher PAC, 24-hour UAldo level, longer duration of hypertension, higher systolic blood pressure, more medications for hypertension and lower serum potassium level and PRA. The serum sodium concentration and 24-hour UNa level were similar between the two groups. The patients with
PA had a thicker LV wall thickness, higher LVMI, and higher inappropriate LVMI compared to the patients with EH (Table 2).

The correlations between 24-hour UAldo level and PAC were moderate in the patients overall, and in the PA and EH groups (Pearson's correlation coefficient: $0.304, \mathrm{p}<0.001 ; 0.215, \mathrm{p}=0.011$; and $0.377, \mathrm{p}<0.001$, respectively). There was no significant correlation between 24-hour UNa level and PAC in the patients overall or in the PA and EH groups. 
Table 2: Echocardiographic parameters of study participants

\begin{tabular}{|c|c|c|c|}
\hline & $\begin{array}{l}\text { Primary aldosteronism } \\
\qquad(\mathrm{n}=158)\end{array}$ & $\begin{array}{c}\text { Essential hypertension } \\
(\mathrm{n}=\mathbf{1 5 8})\end{array}$ & P value \\
\hline Mean wall thickness, mm, mean(SD) & $11.2(1.8)$ & $10.8(1.6)$ & 0.033 \\
\hline LVEDD, mm, mean(SD) & $47(4.6)$ & $46.5(4.9)$ & 0.406 \\
\hline LVESD, mm, mean(SD) & $28.6(3.9)$ & $28.1(4.5)$ & 0.264 \\
\hline LVEDV, mL, mean(SD) & $103.9(24.0)$ & $101.1(26.2)$ & 0.325 \\
\hline LVESV, mL, mean(SD) & $32.1(11.3)$ & $30.9(12.1)$ & 0.403 \\
\hline LVEF, $\%$, mean(SD) & $69.1(6.4)$ & $70.3(6.5)$ & 0.103 \\
\hline LVMI, g/m², mean(SD) & $133.9(37.7)$ & $122.3(36.9)$ & 0.007 \\
\hline Inappropriate LVMI, $\mathrm{g} / \mathrm{m}^{2}$, mean(SD) & $45.6(30.4)$ & $36.7(26.6)$ & 0.007 \\
\hline LVH, $n(\%)$ & $94(59.5 \%)$ & $64(40.5 \%)$ & 0.001 \\
\hline
\end{tabular}

LVEF: left ventricular ejection fraction; LVEDD: left ventricular end-diastolic diameter; LVEDV: left ventricular enddiastolic volume; LVESD: left ventricular end-systolic diameter; LVESV: left ventricular end-systolic volume; LVH: left ventricular hypertrophy: LVMI, left ventricular mass index.

In the patients with PA, the correlations between 24hour UAldo level and LV mass were significant (LVMI $\mathrm{r}=0.231, \mathrm{p}=0.007$; inappropriate LVMI, $\mathrm{r}=0.188, \mathrm{p}=0.029$ ) (Table 3). These correlations remained significant after adjusting for age, sex and systolic blood pressure. The correlations between 24-hour UNa level and LV structural parameters were not significant in the patients with PA. In the patients with $\mathrm{EH}$, the 24-hour UAldo level was not correlated with LV structural parameters, while the 24hour UNa level was correlated with inappropriate LVMI $(\mathrm{r}=0.185, \mathrm{p}=0.027)$. However, the correlations were not statistically significant after adjusting for age, sex and systolic blood pressure (Table 3).

To test if there were non-linear relationships between UNa or UAldo and LVMI, we plotted LVMI according to $\mathrm{UNa}$ or UAldo tertile (Figure $1 \mathrm{~A}$ and $1 \mathrm{~B}$, for the patients with PA; Figure 1C and 1D, for the patient with EH). The LVMI values of the patients with low, medium, and high UNa tertiles were $144.1 \pm 42.9,121.1 \pm$ 33.5 , and $136.7 \pm 32.8 \mathrm{~g} / \mathrm{m}^{2}$, respectively, for the PA group $(\mathrm{p}=0.006$, see also Supplementary Table 2), and $121.2 \pm$ $41.6,113.3 \pm 27.2$ and $129.6 \pm 38.6 \mathrm{~g} / \mathrm{m}^{2}$, respectively, for the EH group ( $\mathrm{p}=0.102)$. The LVMI values of the patients with low, medium, and high UAldo tertiles were $122.1 \pm$ $31.2,129.1 \pm 34.4$, and $146.8 \pm 40.2 \mathrm{~g} / \mathrm{m}^{2}$, respectively, for the PA group ( $\mathrm{p}=0.003)$ (see also Supplementary Table 4 ), and $119.1 \pm 32.7,122.9 \pm 38.6$, and $121.0 \pm 30.6 \mathrm{~g} / \mathrm{m}^{2}$, respectively, for the $\mathrm{EH}$ group $(\mathrm{p}=0.866)$. These results indicated that among patients with PA, the medium UNa tertile group had a lower LVMI compared to the high and low tertile groups. The clinical characters of PA patients with low, medium, and high UNa tertiles were shown in Supplementary Table 1.
Among the patients with PA, multifactor ANOVA revealed significantly different LVMI values in the $\mathrm{UNa}$ and UAldo tertiles after adjusting for covariates (Table 4). There were no interactions between $\mathrm{UNa}$ and UAldo tertiles. Post-hoc analysis with Tukey's honest significant difference test revealed significant differences between the medium and high UNa tertiles and the medium and low tertiles, but not between the low and high tertiles (Supplementary Table 4).

Because LV mass depends on the LV mean wall thickness (MWT) and LV end-diastolic volume (LVEDV), we tested the relationship between these two LV structural parameters and 24-hour UNa or UAldo tertile among the patients with PA. The results showed that MWT and LVEDV were significantly different among the three UNa tertiles after adjusting for age, sex, hypertension duration, blood pressure and tertile of UAldo (Supplementary Table 3). Post-hoc analysis revealed that the LVEDV was significantly higher in the high UNa tertile compared to the medium UNa tertile, while the MWT was significantly higher in the low UNa tertile compared to the medium UNa tertile (Figure 2A and 2C; Supplementary Table 4). In contrast, the MWT and LVEDV were only borderline different among the three UAldo tertiles after adjusting for age, sex, hypertension duration, blood pressure and tertile of UNa (Figure 2B and 2D; Supplementary Table 4).

\section{DISCUSSION}

Our results demonstrated that in patients with PA, the 24-hour UAldo level was positively associated with LVMI, while the medium UNa tertile was associated with a lower LVMI compared to the low or high UNa tertiles. 
Table 3: The correlations between 24-hour urinary sodium or aldosterone and LV structural parameters among (1) patients with primary aldosteronism or (2) patients with essential hypertension

\begin{tabular}{|c|c|c|c|c|c|c|c|c|c|}
\hline \multirow{2}{*}{\multicolumn{2}{|c|}{$\mathbf{L V}$}} & \multicolumn{4}{|c|}{ Primary aldosteronism } & \multicolumn{4}{|c|}{ Essential hypertension } \\
\hline & & \multicolumn{2}{|c|}{ Crude } & \multicolumn{2}{|c|}{ Adjusted* } & \multicolumn{2}{|c|}{ crude } & \multicolumn{2}{|c|}{ Adjusted* } \\
\hline $\begin{array}{l}\text { Structural } \\
\text { parameters }\end{array}$ & & $\begin{array}{c}\text { Correlation } \\
\text { coefficient }\end{array}$ & p-value & $\begin{array}{c}\text { Correlation } \\
\text { coefficient }\end{array}$ & p-value & $\begin{array}{c}\text { Correlation } \\
\text { coefficient }\end{array}$ & p-value & $\begin{array}{c}\text { Correlation } \\
\text { coefficient }\end{array}$ & p-value \\
\hline \multirow[t]{2}{*}{ LVMI } & $\mathrm{UNa}$ & -0.024 & 0.769 & -0.087 & 0.292 & 0.117 & 0.161 & 0.132 & 0.122 \\
\hline & UAldo & 0.231 & 0.007 & 0.216 & 0.013 & 0.074 & 0.368 & 0.078 & 0.351 \\
\hline \multirow[t]{2}{*}{$\begin{array}{l}\text { Inappropriate } \\
\text { LVMI }\end{array}$} & $\mathrm{UNa}$ & -0.035 & 0.668 & -0.097 & 0.239 & 0.185 & 0.027 & 0.141 & 0.097 \\
\hline & UAldo & 0.188 & 0.029 & 0.178 & 0.041 & 0.092 & 0.274 & 0.119 & 0.160 \\
\hline
\end{tabular}

LVMI: left ventricular mass index; UAldo: 24-hour urinary aldosterone amount; UNa: 24-hour urinary sodium amount

*Adjusted by age, sex, systolic blood pressure

Table 4: Multi-factor ANOVA for LVMI by UAldo and UNa tertiles among patients with primary aldosteronism

\begin{tabular}{lcc}
\hline & \multicolumn{3}{c}{$\begin{array}{c}\text { Primary aldosteronism } \\
\mathbf{N}=\mathbf{1 5 8}\end{array}$} \\
\hline Source & F statistic & p-value \\
\hline Age & 0.28 & 0.598 \\
Sex & 13.15 & $<0.001$ \\
Hypertension duration & 1.98 & 0.162 \\
Mean blood pressure & 5.78 & 0.018 \\
UAldo, tertile & 6.11 & 0.003 \\
UNa, tertile & 7.29 & 0.001 \\
UAldo*UNa & 0.25 & 0.907 \\
\hline
\end{tabular}

UAldo: 24-hour urinary aldosterone amount; UNa: 24-hour urinary sodium amount

These differences in LVMI can partly be explained by the LVEDV in the medium versus high UNa tertiles and by the MWT in the low versus medium UNa tertiles.

The relationships between dietary sodium intake, aldosterone and LV dimension have been previously investigated. Pimenta et al recruited 21 patients with PA and 21 patients with EH matched by age, gender and blood pressure, and found that urinary sodium was positively correlated with LV mass in the patients with PA [14]. The difference between their results and ours is probably due to the difference in sodium intake. The urinary sodium level in their PA patients was $216.1 \pm 65.6 \mathrm{mmol} / \mathrm{d}$, compared to $154.4 \pm 91.8 \mathrm{mmol} / \mathrm{d}$ in our PA patients. The urinary sodium level in our study is closer to the world mean dietary sodium level (158 mmol/d) [29] and a prior report in Taiwan (122 mmol/d) [30]. Therefore, their data depicted the relationship between the sodium level among PA patients with higher sodium intake (corresponding to the medium and high tertiles in our data) and LV structure.
One recent study investigated the association between dietary salt intake and cardiac change after treatment in patients with PA [31]. The mean urinary sodium level of patients with PA in that study $(149 \mathrm{mmol} / \mathrm{d})$ is closer to ours. However, the mean urinary sodium of low tertile in our study $(75.8 \mathrm{mmol} / \mathrm{d})$ is much lower than theirs $(100$ $\mathrm{mmol} / \mathrm{d})$. Furthermore, the major LV mass difference in that study is between medium (LV mass index $49.0 \pm$ $9.6 \mathrm{~g} / \mathrm{m}^{2}$ ) and higher tertile (LV mass index $55.9 \pm 14.3 \mathrm{~g} /$ $\mathrm{m}^{2}$ ). There is only minimal difference of LV mass between patients with medium and lower tertile (LV mass index $46.4 \pm 7.3 \mathrm{~g} / \mathrm{m}^{2}$ ). Our results expand the current knowledge about the relationship between the sodium level in PA patients and a wider range of sodium intake and LV structure.

The finding that a medium tertile of sodium intake was associated with a lower LV mass has not been reported before in patients with PA and EH. However, U- or J-shaped relationships between sodium intake 

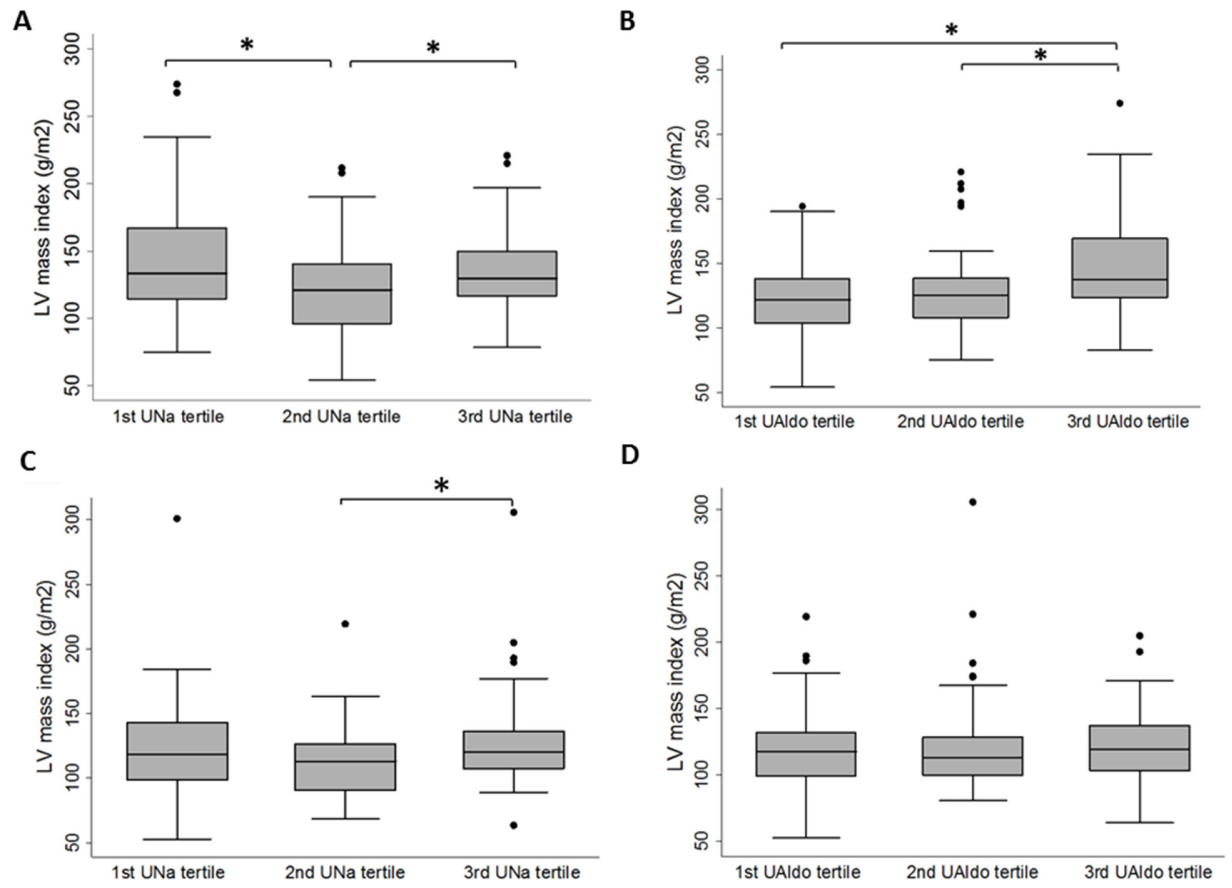

Figure 1: Box plots of left ventricular (LV) mass index by 24-hour urinary sodium (UNa) tertiles or 24-hour urinary aldosterone (Ualdo) tertiles among patients with primary aldosteronism $(\mathbf{A}, \mathbf{B})$ or essential hypertension $(\mathbf{C}$, D). The thick horizontal line represents the median LV mass index, the box represents interquartile range, whiskers represent $95 \%$ confidence intervals, and dots represent outlying observations. ${ }^{*} \mathrm{p}<0.05$ in post-hoc analysis.

A

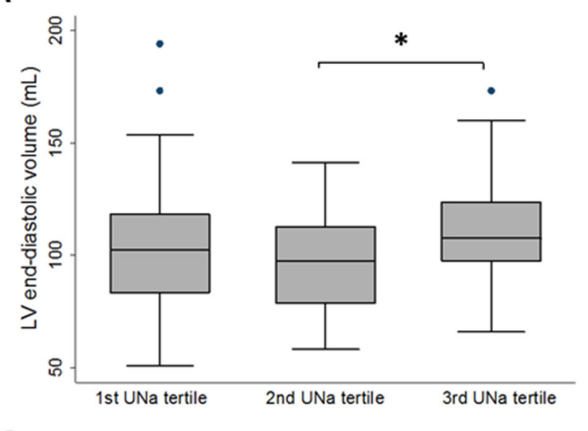

C

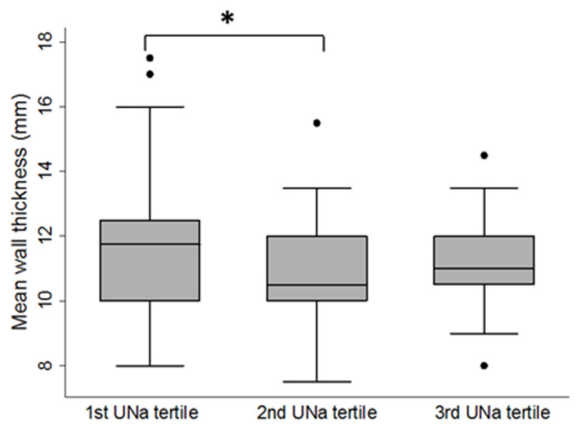

B

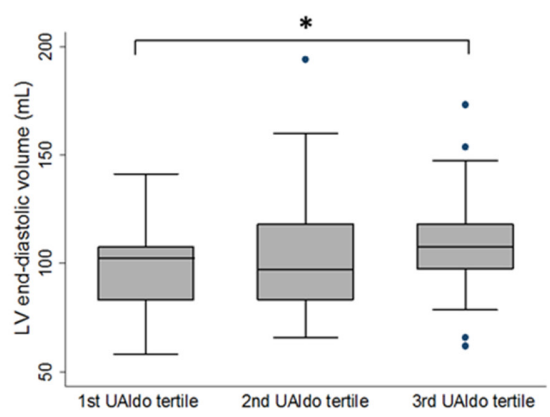

D

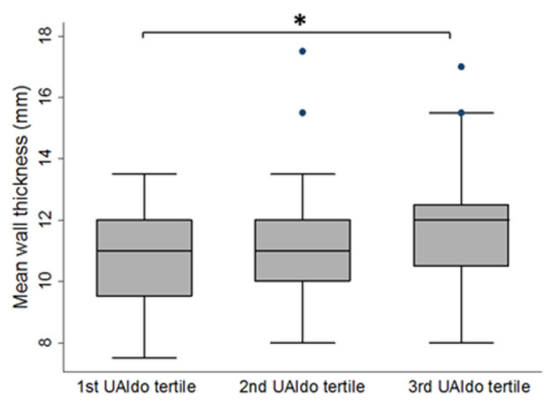

Figure 2: Box plots of left ventricular (LV) end-diastolic volume or mean wall thickness by 24-hour urinary sodium (UNa) tertiles or 24-hour urinary aldosterone (Ualdo) tertiles among patients with primary aldosteronism. (A) LV enddiastolic volume, by 24-hour UNa tertile. (B) LV end-diastolic volume, by 24-hour UAldo tertile. (C) Mean wall thickness, by 24-hour UNa tertile. (D) Mean wall thickness, by 24-hour UAldo tertile. The thick horizontal line represents the mean value, the box represents interquartile range, whiskers represent $95 \%$ confidence intervals, and dots represent outlying observations. $* \mathrm{p}<0.05$ in post-hoc analysis. 
and hypertension or cardiovascular events have been reported. In a prior study in Taiwan, 24-hour urinary sodium level was curvilinearly associated with incident hypertension among 1520 middle-aged and elderly participants during 7.9 year of follow-up [30]. The participants with a urinary sodium level between 84-122 $\mathrm{mmol} /$ day had the lowest rate of incident hypertension compared to the participants with lower or higher sodium levels. In the Prospective Urban Rural Epidemiology (PURE) study, which included 101,945 participants, a J-shaped association between sodium intake and cardiovascular disease or death was demonstrated [16]. A recent pooled analysis from four large prospective studies also supported this finding [32]. Although these studies were carefully conducted, a reverse causation cannot be fully excluded. A prior pre-clinical study reported that low dietary sodium intake can still induce LV hypertrophy and fibrosis in the presence of excess aldosterone. Hattori et al used uninephrectomized rats with low or high salt intake and aldosterone minipump implantation to investigate cardiac damage [17]. The results showed that in the setting of low salt intake and excess aldosterone, substantial cardiac remodeling and diastolic dysfunction occurred. In addition, blood pressure was not elevated in the low salt intake group, although the concentration of reactive oxygen species was significantly increased. These findings imply that excess levels of aldosterone can induce hypertrophy and fibrosis in low-salt conditions via inflammation without the contribution of hypertension. Nevertheless, low salt intake has been associated with elevated plasma catecholamine and aldosterone concentrations among patients with EH $[33,34]$. Further studies are warranted to investigate whether an elevated level of catecholamine contributes to a higher LV mass in patients with PA with lower salt intake.

Our study revealed that the increased LVMI in high UNa tertile was associated with increased LVEDV and increased LVMI in low UNa tertile was associated with increased MWT in PA patients. The detail mechanisms whether salt/aldosterone combination promotes cardiac dimension and structure change are still unclear. Both aldosterone and high salt intake induce fluid retention and expand intravascular volume [14]. In the study by Pimeta et al, PA patients have much higher LVEDV than $\mathrm{EH}$ patients $(115.1 \pm 29.3 \mathrm{ml}$ for PA patients vs. $80.6 \pm 22.2 \mathrm{ml}$ for $\mathrm{EH}$ patients, $\mathrm{p}<0.001$ ) [14]. However, PA patients also have much higher UNa value than $\mathrm{EH}$ patients $(216.1 \pm 65.6 \mathrm{mmol} / \mathrm{d}$ for PA patients vs.155.5 $\pm 65.6 \mathrm{mmol} / \mathrm{d}$ for $\mathrm{EH}$ patients, $\mathrm{p}=0.004)$. In that study, high salt intake in hyperaldosterone status leads to the great difference of LVEDV between PA and EH patients. In the presented study, PA patients with high Una tertile had higher LVEDV than PA patients with medium UNa tertile, which also demonstrate the effect of high salt intake on LVEDV in hyperaldosterone status. In the other aspect, increased LVMI in low UNa tertile was associated with increased MWT in PA patients in the presented study. Reduced salt intake is associated with increased plasma norepinephrine concentrations, and the effects are more stronger in hypertensive patients [33, 34]. In addition, elevated plasma norepinephrine concentrations are associated with increased LV wall thickness and concentric LV hypertrophy [35]. Therefore, increased plasma norepinephrine concentrations may be one of the reasons to explain the increased MWT in PA patients with low UNa tertile.

The relationship between sodium intake and LVMI may also be modified by genetic factors. In a previous clinical study evaluating the relationship between angiotensin-converting enzyme (ACE) genotypes and LVMI, a medium sodium intake was associated with a lower LVMI compared to a higher or lower sodium intake in Italian offspring with ACE DD and DI genotypes but not in the II genotype or in Slavic offspring [36]. In the study by Isaji et al, there is a significant correlation between urinary sodium and LVMI in patients with CYP11B2 C/C, but not in CYP11B2 C/T and CYP11B2 $\mathrm{T} / \mathrm{T}$ [37]. However, the incidence of $\mathrm{CYP} 11 \mathrm{~B} 2 \mathrm{C} / \mathrm{C}$ is only $7.5 \%$ in Taiwanese [38]. This might also weaken the relation between UNa and LVMI in the presented study.

There are several limitations to this study. First, we use a single 24-hour urine collection to estimate dietary salt as previous studies [14, 39]. However, regarding the day-to-day variability of salt intake, the average derived from multiple measurement of 24-hour urinary sodium excretion are more accurate compared with a single 24hour measurement. The variability in the urinary sodium measurement may underestimate the association between sodium intake and cardiac dimensions. Second, some anti-hypertensive medication may have influence on left ventricular structure and urinary sodium excretion. Further studies are need to clarify their effects. Third, because of the cross-sectional design, a clear causation could not be established based on our results. Therefore, recommendations on dietary salt intake in patient with PA cannot be given based on our results. Fourth, compared with other population-based studies in patients with EH, our sample size was relatively small, and this may have prevented us from showing a relationship between LVMI and urinary sodium or aldosterone. Further large-scale studies are needed to confirm our results. Last, the duration of hypertension was different between PA and EH groups. Further studies with same hypertension duration in both groups are needed in the future.

\section{CONCLUSION}

In the patients with PA, a medium 24-hour UNa level was associated with a lower LVMI compared to a higher or lower UNa level, and the 24-hour UAldo level was positively associated with LVMI. 


\section{Appendix}

Membership of the Taiwan Primary Aldosteronism Investigation (TAIPAI) Study Group: Che-Hsiung Wu, MD (Chi-Taz hospital, PI of Committee); Vin-Cent Wu, $\mathrm{MD}, \mathrm{PhD}$ (NTUH, PI of Committee); Yen-Hung Lin, MD, $\mathrm{PhD}$ (NTUH, PI of Committee); Yi-Luwn Ho, MD, PhD (NTUH, PI of Committee); Hung-Wei Chang, MD, PhD (Far Eastern Hospital, PI of Committee); Lian-Yu Lin MD, $\mathrm{PhD}$ (NTUH, PI of Committee); Fu-Chang Hu, MS, ScD, (Harvard Statistics, Site Investigator); Kao-Lang Liu, MD (NTUH, PI of Committee); Shuo-Meng Wang, MD (NTUH, PI of Committee); Kuo-How Huang, MD, PhD (NTUH, PI of Committee); Yung-Ming Chen, MD (NTUH, PI of Committee); Chin-Chi Kuo; MD (Yun-Lin, PI of Committee), Chin-Chen Chang, MD (NTUH, PI of Committee); ShihCheng Liao, MD (NTUH, PI of Committee); Ruoh-Fang Yen, MD, PhD (NTUH, PI of Committee); and Kwan-Dun $\mathrm{Wu}, \mathrm{MD}, \mathrm{PhD}$ (NTUH, Director of Coordinating Center).

\section{ACKNOWLEDGMENTS}

The authors thank Professor Michael Stowasser for valuable scientific comments on the manuscript and the staff of the $2^{\text {nd }}$ and the $3^{\text {rd }}$ core facility at National Taiwan University Hospital for technical assistance and facility support. This study was supported by the TaTung Kidney Foundation, National Taiwan University Hospital (NTUH 105-S3044, NTUH 106-S3406, NTUH 106-S3471, UN105-060), National Taiwan University Hospital Hsin-Chu Branch (105-HCH035), Ministry of Science and Technology (MOST 105-2314-B-002-122MY3, MOST 105-2314-B-002-123), and Department of Health, Executive Yuan, R.O.C. (DOH 104-HO-1012, PTH-10508). The funders had no role in study design, data collection and analysis, decision to publish, or preparation of the manuscript.

\section{CONFLICTS OF INTEREST}

The authors declare no competing interests.

\section{REFERENCES}

1. Schirpenbach C, Reincke M. Primary aldosteronism: current knowledge and controversies in Conn's syndrome. Nat Clin Pract Endocrinol Metab. 2007; 3:220-227.

2. Young WF Jr. Minireview: primary aldosteronismchanging concepts in diagnosis and treatment. Endocrinology. 2003; 144:2208-2213.

3. Savard S, Amar L, Plouin PF, Steichen O. Cardiovascular complications associated with primary aldosteronism: a controlled cross-sectional study. Hypertension. 2013; 62:331-336.
4. Rossi GP, Sacchetto A, Pavan E, Palatini P, Graniero GR, Canali C, Pessina AC. Remodeling of the left ventricle in primary aldosteronism due to Conn's adenoma. Circulation. 1997; 95:1471-1478.

5. Tanabe A, Naruse M, Naruse K, Hase M, Yoshimoto T, Tanaka M, Seki T, Demura R, Demura H. Left ventricular hypertrophy is more prominent in patients with primary aldosteronism than in patients with other types of secondary hypertension. Hypertens Res. 1997; 20:85-90.

6. Shigematsu Y, Hamada M, Okayama H, Hara Y, Hayashi Y, Kodama K, Kohara K, Hiwada K. Left ventricular hypertrophy precedes other target-organ damage in primary aldosteronism. Hypertension. 1997; 29:723-727.

7. Levy D, Garrison RJ, Savage DD, Kannel WB, Castelli WP. Prognostic implications of echocardiographically determined left ventricular mass in the Framingham Heart Study. N Engl J Med. 1990; 322:1561-1566.

8. Muiesan ML, Salvetti M, Paini A, Agabiti-Rosei C, Monteduro C, Galbassini G, Belotti E, Aggiusti C, Rizzoni D, Castellano M, Agabiti-Rosei E. Inappropriate left ventricular mass in patients with primary aldosteronism. Hypertension. 2008; 52:529-534.

9. Connell JM, MacKenzie SM, Freel EM, Fraser R, Davies E. A lifetime of aldosterone excess: long-term consequences of altered regulation of aldosterone production for cardiovascular function.Endocr Rev. 2008; 29:133-154.

10. Briet M, Schiffrin EL. Aldosterone: effects on the kidney and cardiovascular system. Nat Rev Nephrol. 2010; 6:261-273.

11. Rossi GP, Cesari M, Cuspidi C, Maiolino G, Cicala MV, Bisogni V, Mantero F, Pessina AC. Response to effectiveness of adrenalectomy and aldosterone antagonists for long-term treatment of primary aldosteronism. Hypertension. 2013; 62:e14.

12. Jin Y, Kuznetsova T, Maillard M, Richart T, Thijs L, Bochud M, Herregods MC, Burnier M, Fagard R, Staessen JA. Independent relations of left ventricular structure with the 24-hour urinary excretion of sodium and aldosterone. Hypertension. 2009; 54:489-495.

13. Schmieder RE, Messerli FH, Garavaglia GE, Nunez BD. Dietary salt intake. A determinant of cardiac involvement in essential hypertension. Circulation. 1988; 78:951-956.

14. Pimenta E, Gordon RD, Ahmed AH, Cowley D, Leano R, Marwick TH, Stowasser M. Cardiac dimensions are largely determined by dietary salt in patients with primary aldosteronism: results of a case-control study. J Clin Endocrinol Metab. 2011; 96:2813-2820.

15. McCarron DA, Kazaks AG, Geerling JC, Stern JS, Graudal NA. Normal range of human dietary sodium intake: a perspective based on 24-hour urinary sodium excretion worldwide. Am J Hypertens. 2013; 26:1218-1223.

16. O’Donnell M, Mente A, Rangarajan S, McQueen MJ, Wang X, Liu L, Yan H, Lee SF, Mony P, Devanath A, Rosengren A, Lopez-Jaramillo P, Diaz R, et al. Urinary sodium and 
potassium excretion, mortality, and cardiovascular events. N Engl J Med. 2014; 371:612-623.

17. Hattori T, Murase T, Sugiura Y, Nagasawa K, Takahashi K, Ohtake M, Ohtake M, Miyachi M, Murohara T, Nagata K. Effects of salt status and blockade of mineralocorticoid receptors on aldosterone-induced cardiac injury. Hypertens Res. 2014; 37:125-133.

18. Lin YH, Lee HH, Liu KL, Lee JK, Shih SR, Chueh SC, Lin WC, Lin LC, Lin LY, Chung SD, Wu VC, Kuo CC, Ho YL, et al. Reversal of myocardial fibrosis in patients with unilateral hyperaldosteronism receiving adrenalectomy. Surgery. 2011; 150:526-533.

19. Wu KD, Liao TS, Chen YM, Lai MK, Chen SJ, Su CT, Chu TS, Chang CC, Hsieh BS. Preoperative diagnosis and localization of aldosterone-producing adenoma by adrenal venous sampling after administration of metoclopramide. J Formos Med Assoc. 2001; 100:598-603.

20. Wu VC, Yang SY, Lin JW, Cheng BW, Kuo CC, Tsai CT, Chu TS, Huang KH, Wang SM, Lin YH, Chiang CK, Chang HW, Lin CY, et al. Kidney impairment in primary aldosteronism. Clin Chim Acta. 2011; 412:1319-1325.

21. Chao CT, Wu VC, Kuo CC, Lin YH, Chang CC, Chueh SJ, Wu KD, Pimenta E, Stowasser M. Diagnosis and management of primary aldosteronism: an updated review. Ann Med. 2013; 45:375-383.

22. TAIPAI Study Group, Wu VC, Chueh SC, Chang HW, Lin LY, Liu KL, Lin YH, Ho YL, Lin WC, Wang SM, Huang $\mathrm{KH}$, Hung KY, Kao TW, et al. Association of kidney function with residual hypertension after treatment of aldosteroneproducing adenoma. Am J Kidney Dis. 2009; 54:665-673.

23. Wu VC, Chao CT, Kuo CC, Lin YH, Chueh SC, Wu KD. Diagnosis and management of primary aldosteronism. Acta Nephrologica. 2012; 26:111-120.

24. Kuo CC, Wu VC, Huang KH, Wang SM, Chang CC, Lu CC, Yang WS, Tsai CW, Lai CF, Lee TY, Lin WC, Wu MS, Lin $\mathrm{YH}$, et al. Verification and evaluation of aldosteronism demographics in the Taiwan Primary Aldosteronism Investigation Group (TAIPAI Group). J Renin Angiotensin Aldosterone Syst. 2011; 12:348-357.

25. Lang RM, Bierig M, Devereux RB, Flachskampf FA, Foster E, Pellikka PA, Picard MH, Roman MJ, Seward J, Shanewise JS, Solomon SD, Spencer KT, Sutton MS, et al. Recommendations for chamber quantification: a report from the American Society of Echocardiography's Guidelines and Standards Committee and the Chamber Quantification Writing Group, developed in conjunction with the European Association of Echocardiography, a branch of the European Society of Cardiology. J Am Soc Echocardiogr. 2005; 18:1440-1463.

26. Devereux RB, Reichek N. Echocardiographic determination of left ventricular mass in man. Anatomic validation of the method. Circulation. 1977; 55:613-618.

27. de Simone G, Devereux RB, Kimball TR, Mureddu GF, Roman MJ, Contaldo F, Daniels SR. Interaction between body size and cardiac workload: influence on left ventricular mass during body growth and adulthood. Hypertension. 1998; 31:1077-1082.

28. Devereux RB. Detection of left ventricular hypertrophy by M-mode echocardiography. Anatomic validation, standardization, and comparison to other methods. Hypertension. 1987; 9:II19-26.

29. Hung CS, Ho YL, Chang YY, Wu VC, Wu XM, Lee JK, Chueh SC, Lin YH, Changh YS, Yang SY, Hu YH, Sui MJ, Chen MF, Wu KD. Twenty-four-hour urinary aldosterone predicts inappropriate left ventricular mass index in patients with primary aldosteronism. ScientificWorldJournal. 2013; 2013:294594.

30. Chien KL, Hsu HC, Chen PC, Su TC, Chang WT, Chen MF, Lee YT. Urinary sodium and potassium excretion and risk of hypertension in Chinese: report from a community-based cohort study in Taiwan. J Hypertens. 2008; 26:1750-1756.

31. Catena C, Colussi G, Novello M, Verheyen ND, Bertin N, Pilz S, Tomaschitz A, Sechi LA. Dietary salt intake is a determinant of cardiac changes after treatment of primary aldosteronism: a prospective study. Hypertension. 2016; 68:204-212.

32. Mente A, O'Donnell M, Rangarajan S, Dagenais G, Lear S, McQueen M, Diaz R, Avezum A, Lopez-Jaramillo P, Lanas F, Li W, Lu Y, Yi S, et al. Associations of urinary sodium excretion with cardiovascular events in individuals with and without hypertension: a pooled analysis of data from four studies. Lancet. 2016; 388:465-475.

33. Graudal NA, Galloe AM, Garred P. Effects of sodium restriction on blood pressure, renin, aldosterone, catecholamines, cholesterols, and triglyceride: a metaanalysis. JAMA. 1998; 279:1383-1391.

34. Graudal NA, Hubeck-Graudal T, Jurgens G. Effects of lowsodium diet vs. high-sodium diet on blood pressure, renin, aldosterone, catecholamines, cholesterol, and triglyceride (Cochrane Review). Am J Hypertens. 2012; 25:1-15.

35. Zoccali C, Mallamaci F, Tripepi G, Parlongo S, Cutrupi S, Benedetto FA, Cataliotti A, Malatino LS, investigators C. Norepinephrine and concentric hypertrophy in patients with end-stage renal disease. Hypertension. 2002; 40:41-46.

36. Kuznetsova T, Staessen JA, Stolarz K, Ryabikov A, Tikhonoff V, Olszanecka A, Bianchi G, Brand E, Casiglia E, Dominiczak A, Fagard R, Malyutina S, Nikitin Y, et al. Relationship between left ventricular mass and the ACE $\mathrm{D} / \mathrm{I}$ polymorphism varies according to sodium intake. J Hypertens. 2004; 22:287-295.

37. Isaji M, Mune $\mathrm{T}$, Takada N, Yamamoto $\mathrm{Y}$, Suwa $\mathrm{T}$, Morita H, Takeda J, White PC. Correlation between left ventricular mass and urinary sodium excretion in specific genotypes of CYP11B2. J Hypertens. 2005; 23:1149-1157.

38. Chou $\mathrm{CH}$, Ueng $\mathrm{KC}$, Yang $\mathrm{SF}$, Wu $\mathrm{CH}$, Wang $\mathrm{PH}$. Relationship of genetic polymorphisms of aldosterone 
synthase gene cytochrome p450 11b2 and mineralocorticoid receptors with coronary artery disease in Taiwan. Int J Med Sci. 2016; 13:117-123.

39. Gaddam K, Corros C, Pimenta E, Ahmed M, Denney T, Aban I, Inusah S, Gupta H, Lloyd SG, Oparil S,
Husain A, Dell'Italia LJ, Calhoun DA. Rapid reversal of left ventricular hypertrophy and intracardiac volume overload in patients with resistant hypertension and hyperaldosteronism: a prospective clinical study. Hypertension. 2010; 55:1137-1142. 